ESAIM: PROCEEDINGS, August 2010, Vol. 30, p. 104-123

D. Bresch, V. Calvez, E. Grenier, P. Vigneaux \& J.-F. Gerbeau, Editors

\title{
SIMULATION OF SELF-PROPELLED CHEMOTACTIC BACTERIA IN A STOKES FLOW*
}

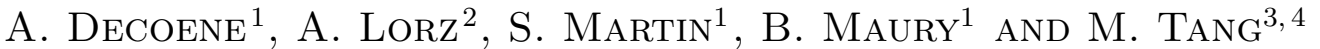

\begin{abstract}
We present a method to simulate the motion of self-propelled rigid particles in a twodimensional Stokesian fluid, taking into account chemotactic behaviour. Self-propulsion is modelled as a point force associated to each particle, placed at a certain distance from its gravity centre. The method for solving the fluid flow and the motion of the bacteria is based on a variational formulation on the whole domain, including fluid and particles: rigid motion is enforced by penalizing the strain rate tensor on the rigid domain, while incompressibility is treated by duality. This leads to a minimization problem over unconstrained functional spaces which can be easily implemented from any finite element Stokes solver. In order to ensure robustness, a projection algorithm is used to deal with contacts between particles. The particles are meant to represent bacteria of the Escherichia coli type, which interact with their chemical environment through consumption of nutrients and orientation in some favorable direction. Our model takes into account the interaction with oxygen. An advection-diffusion equation on the oxygen concentration is solved in the fluid domain, with a source term accounting for oxygen consumption by the bacteria. In addition, self-propulsion is deactivated for those particles which cannot consume enough oxygen. Finally, the model includes random changes in the orientation of the individual bacteria, with a frequency that depends on the surrounding oxygen concentration, in order to favor the direction of the concentration gradient and thus to reproduce chemotactic behaviour. Numerical simulations implemented with FreeFem ++ are presented.
\end{abstract}

* This work has been supported by the ANR project MOSICOB "Modélisation et Simulation de Fluides Complexes Biomimétiques".

${ }^{1}$ Université Paris-Sud 11, Département de Mathématiques, Bâtiment 425, 91405 Orsay cedex (France)

2 Department of Applied Mathematics and Theoretical Physics, University of Cambridge, Wilberforce Road, CB3 0WA (UK)

${ }^{3}$ Université Pierre \& Marie Curie, Laboratoire Jacques-Louis Lions, Boîte courrier 187, 75252 Paris cedex 05 (France)

${ }^{4}$ INRIA Paris-Rocquencourt, BANG Project, Domaine de Voluceau, BP 105, 78153 Rocquencourt (France)

(C) EDP Sciences, SMAI 2010 


\begin{abstract}
Résumé. Nous présentons une méthode de simulation du mouvement de particules rigides autopropulsées dans un fluide de Stokes en dimension 2. en prenant en compte leur comportement chimiotactique. L'auto-propulsion est modélisée par une force (presque) ponctuelle associée à chaque particule et placée à une certaine distance de son centre de gracité. La méthode de résolution de l'écoulement fluide et du mouvement des bactéries est basée sur une formulation variationnelle dans tout le domaine, incluant le fluide et les les particules : le mouvement rigide des particules est obtenu par pénalisation du tenseur de déformation sur le domaine rigide, alors que l'incompressibilité du fluide est traitée par dualité. Cela conduit à un problème de minimisation sur un espace fonctionnel non contraint qui peut être facilement implanté à partir d'un solveur de Stokes de type Éléments Finis. Afin d'assurer la robustesse de la méthode, un algorithme de projection est utilisé afin de traiter le contact des particules rigides. Les particules sont supposées représenter des bactéries du type Escherichia coli, qui interagissent avec leur environnement chimique à travers la consommation de nutriments et l'orientation vers des directions favorables à cette consommation. Notre modèle prend en compte l'interaction avec l'oxygène : une équation d'advection-diffusion de la concentration d'oxygène est résolue dans le domaine, avec un terme source modélisant la consommation de l'oxygène par les bactéries. De plus, l'auto-propulsion est désactivée pour les bactéries qui ne consomment qu'une faible quantité d'oxygène. Finalement, le modèle inclut des changements de direction aléatoires de la part de chaque individu, avec une fréquence qui dépend de la concentration d'oxygène locale, afin de favoriser la direction du gradient de concentration et, ainsi, de reproduire le comportement chimiotactique des bactéries. Des simulations numériques implantées avec FreeFem++ sont présentées.
\end{abstract}

\title{
INTRODUCTION
}

Some bacteria, like Escherichia coli and Bacillus subtilis, are able to swim in a viscous fluid by means of self-propulsion. Although living at low Reynolds regime $(R e \ll 1)$, they are capable of swimming many body lengths per second [4]. As described by Berg [1], individual cells of these bacteria are rod-shaped (length about $2 \mu \mathrm{m}$, diameter of the order of $1 \mu \mathrm{m}$ ), and their surface is flagellated: the helical flagella, responsible for propulsion, are distributed randomly over their cell body, emerging from motors that are fixed within the cell membrane (see [21] and [5]). When the motor turns counter-clockwise, the filaments wrap into a bundle that pushes the cell along at speeds of 25 to $35 \mu \mathrm{m} \mathrm{s}^{-1}$. When one or more of the motors reverse, the swimming direction of the cell can change. Indeed, it is well known (see $[1,5,19]$ ) that the bacteria we consider alternate two basic behavioral modes: a more or less linear motion called a run, and a highly erratic motion called tumble, the purpose of which is to reorient the cell. During a run the bacterium moves in the most recently chosen direction. Then it tumbles, i.e. it randomly chooses a new direction and starts moving straightforward again. That is how E. Coli performs chemotaxis, which means swimming up a chemoattractant gradient. Run times are typically much longer than the time spent tumbling, and they are closely related to the surrounding environment. In fact, when bacteria move in a favorable direction (i.e. in the direction of a nutrient concentration gradient) the run times are increased; on the contrary, they are decreased when the direction is not favorable. These bacteria are too small to detect instantaneously spatial differences in the concentration of an attractant at their own length scale. Therefore they measure if they are going in the right direction by doing several successive evaluations of the concentration in time. The effect of alternating these two modes of behavior, and in particular, of increasing the run length when moving in a favorable direction, is that a bacterium executes a three-dimensional random walk with drift in a favorable direction when observed on a sufficiently long time scale.

We aim at modelling collective phenomena of (possibly dense) suspensions, by means of direct numerical simulations. As suggested in [2], bacteria are modelled as spherical particles of constant radius, each of which has an associated localized force, representing the propulsion force exerted by the flagellum. The action of the flagellum is modelled by a point force, directed radially outward from the center of the particle with some orientation angle that defines the direction of motion. Since bacteria live in flows at low Reynolds number, 
we consider Stokes flow. This model leads to a coupled problem, composed by the Stokes equations inside the fluid, with no-slip boundaries on the particle boundaries, and the equilibrium of forces on each rigid particle. We follow the method used in [12] and [15] to simulate rigid particles in a fluid flow. This method is based on a variational formulation on the whole domain, including fluid and particles. Rigid motion is enforced by penalizing the strain rate tensor on the rigid domain, while incompressibility is treated by duality. This leads to a minimization problem over unconstrained functional spaces which can be easily implemented from any finite element Stokes solver. In order to ensure robustness, an efficient algorithm is used to deal with contacts between particles.

We also simulate the interaction of bacteria with oxygen. For that purpose we take into account various phenomena. The oxygen concentration is advected by the fluid and diffuses. Cell respiration is taken into account through a sink term in the advection-diffusion equation, that leads to a decrease of the oxygen concentration in the zones occupied by the bacteria. In addition, the intensity of the self-propulsion force vanishes when a bacterium is not provided with enough oxygen. Finally, individual tumbling frequencies decrease when the bacteria are swimming towards higher oxygen concentrations and decrease in the opposite case, so that the direction of the concentration gradient is favored at the macroscopic level.

The simulations presented in this work have been implemented with FreeFem $++[9]$.

\section{THE MODEL}

\subsection{Hydrodynamic effects and self-propulsion}

We consider a connected bounded and regular domain $\Omega \subset \mathbb{R}^{2}$ and we denote by $\left(B_{i}\right)_{i=1, \ldots, N}$ the rigid particles, strongly included in $\Omega$ (see right in Fig 1). $B$ denotes the whole rigid domain: $B=\cup_{i} B_{i}$. The domain $\Omega \backslash \bar{B}$ is filled with Newtonian fluid governed by the Stokes equations. Here, $\mu$ is the viscosity of the fluid and $\mathbf{f}_{f}$ the external forces exerted on it. Since we consider a Newtonian fluid, the stress tensor $\underline{\underline{\sigma}}$ writes

$$
\underline{\underline{\sigma}}=2 \mu \mathbb{D}(\mathbf{u})-p \mathbb{I}, \quad \text { where } \quad \mathbb{D}(\mathbf{u})=\frac{\nabla \mathbf{u}+(\nabla \mathbf{u})^{T}}{2}
$$

For the sake of simplicity we will consider homogeneous Dirichlet conditions on $\partial \Omega$. On the other hand, viscosity imposes a no-slip condition on the boundary $\partial B$ of the rigid domain.

Following [2], bacteria are modeled as spherical particles of constant radius $R_{b}$, each of which has an associated point force, representing the force exerted by the flagella on the fluid. A force of same intensity but opposite sign is exerted on the particle, due to propulsion. Actually, in order to regularize the problem, the force applied by the flagellar bundle on the fluid is represented by a volume force density $\mathbf{f}_{p}$ supported in a ball $P$ of small radius $R_{p}$ and which center is placed at some constant distance $d$ from the center of sphere $B$. The propulsion force is directed radially outward from the center of the particle and has some orientation angle $\theta$, measured from the $x$-axis, as shown in Fig. 1.

The total force exerted on the fluid by the flagellar bundle associated to the $i$ th particle has a constant magnitude $f_{p}$ and will be denoted by $\mathbf{F}_{p}^{i}$ :

$$
\mathbf{F}_{p}^{i}=-f_{p} \boldsymbol{\tau}_{i}=\int_{P_{i}} \mathbf{f}_{P}^{i} \mathrm{~d} \mathbf{x}, \quad \text { with } \quad \mathbf{f}_{p}^{i}=-\frac{f_{p}}{\operatorname{meas}\left(P_{i}\right)} \boldsymbol{\tau}_{i}
$$



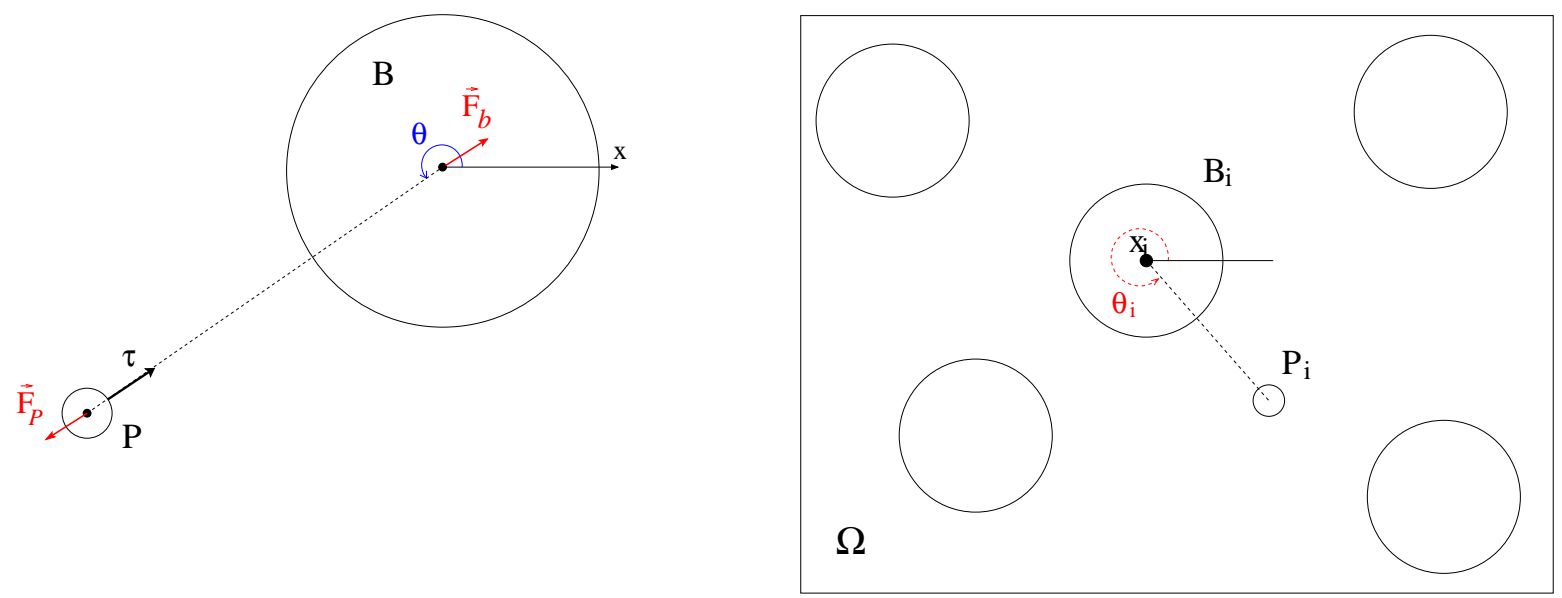

FIGURE 1. Left: single bacterium, right: whole domain with inclusions

where $\boldsymbol{\tau}_{i}$ is given by the orientation angle $\theta_{i}$. The total force exerted by the flagella on their associated particle $B_{i}$ will be denoted by $\mathbf{F}_{b}^{i}$ and is equal to $-\mathbf{F}_{p}^{i}$, so that we can write:

$$
\mathbf{F}_{b}^{i}=f_{p} \boldsymbol{\tau}_{i}=\int_{B_{i}} \mathbf{f}_{b}^{i} \mathrm{~d} \mathbf{x}, \quad \text { with } \quad \mathbf{f}_{b}^{i}=\frac{f_{p}}{\operatorname{meas}\left(B_{i}\right)} \boldsymbol{\tau}_{i} .
$$

At the initial time the particles are distributed randomly over the fluid (without overlapping). The position of the center of the $i$ th particle is denoted by $\mathbf{x}_{i}, \mathbf{v}_{i}$ and $\omega_{i}$ its translational and angular velocities.

We have to find the velocity $\mathbf{u}=\left(u_{1}, u_{2}\right)$ and the pressure field $p$ defined in $\Omega \backslash \bar{B}$, as well as the velocities of the particles $\mathbf{V}:=\left(\mathbf{v}_{i}\right)_{i=1, \ldots, N} \in \mathbb{R}^{2 N}$ and $\boldsymbol{\omega}:=\left(\omega_{i}\right)_{i=1, \ldots, N} \in \mathbb{R}^{N}$ such that:

$$
\left\{\begin{aligned}
-\mu \Delta \mathbf{u}+\nabla p & =\mathbf{f}_{f} & & \text { in } \Omega \backslash \bar{B}, \\
\nabla \cdot \mathbf{u} & =0 & & \text { in } \Omega \backslash \bar{B}, \\
\mathbf{u} & =0 & & \text { on } \partial \Omega, \\
\mathbf{u} & =\mathbf{v}_{i}+\omega_{i} \times\left(\mathbf{x}-\mathbf{x}_{i}\right) & & \text { on } \partial B_{i}, \quad \forall i \in\{1, \ldots, N\} .
\end{aligned}\right.
$$

The external forces considered on the fluid are gravity, and the forces exerted by the flagella, so that

$$
\mathbf{f}_{f}=\sum_{i=1 \ldots N} \mathbf{f}_{p}^{i} \chi_{p}^{i}
$$

where $\chi_{p}^{i}$ is the characteristic function associated to the ball $P_{i}$. Finally, Newton's second law of motion, written here in the non-inertial regime, couples the equations:

$$
\left\{\begin{aligned}
\int_{B_{i}} \mathbf{f}_{b}^{i}-\int_{\partial B_{i}} \underline{\underline{\sigma}} \cdot \mathbf{n} & =0 \quad \forall i, \\
\int_{B_{i}}\left(\mathbf{x}-\mathbf{x}_{i}\right) \times \mathbf{f}_{b}^{i}-\int_{\partial B_{i}}\left(\mathbf{x}-\mathbf{x}_{i}\right) \times \underline{\underline{\sigma}} \cdot \mathbf{n} & =0 \quad \forall i .
\end{aligned}\right.
$$

The motion of each bacterium $B_{i}$ is then set by its instantaneous velocity $\mathbf{u}(\mathbf{x}, t)=\mathbf{v}_{i}(t)+\omega_{i}(t) \times\left(\mathbf{x}-\mathbf{x}_{i}(t)\right)$ defined on $\partial B_{i}$. On one hand, the center $\mathbf{x}_{i}$ of the particle $B_{i}$ follows the differential equation

$$
\dot{\mathbf{x}}_{i}(t)=\mathbf{v}_{i}(t)
$$


On the other hand the relative position of its corresponding flagellum $P_{i}$ rotates with angular velocity $\omega_{i}$, i.e.

$$
\dot{\theta}_{i}(t)=\omega_{i}(t)
$$

\subsection{Tumbling and chemotaxis}

E. Coli performs chemotaxis by alternating running and tumbling, and biasing the duration of the runs. The run durations are exponentially distributed with a rate of average $\lambda \sim 1 \mathrm{~s}^{-1}$ (see [19]). Therefore, we model tumbling through a Poisson process with variable intensity. The time spent tumbling is neglected, and the run time $T_{\text {run }}$ of each bacterium, i.e. the time elapsed between two tumbles, follows an exponential law of parameter $\lambda$, which represents the tumbling frequency. This frequency varies depending on the surrounding environment of the bacterium, that is depending on if it is going in a favorable direction or not.

Let $c$ be the concentration of the chemical attractant considered. A first simplified model for the tumbling frequency reads as:

$$
\lambda(c)=1-\varepsilon \phi\left(\frac{\mathrm{D} c}{\mathrm{D} t}\right)
$$

were $\mathrm{D} c / \mathrm{D} t$ is the Lagrangian derivative of $c$ along the trajectory of the bacterium, and $\phi$ is the sign function. We could also use a model of the following, more realistic type, used in several earlier models (see for example [19]):

$$
\lambda(c)=1-\varepsilon \phi\left(\int_{t-\Delta t}^{t} c(s) g(t-s) \mathrm{d} s\right)
$$

where $g(s)=1$ if $s \leq \Delta t / 4$ and $g(s)=-1 / 3$ if $s>\Delta t / 4$. The latter model assumes that the bacterium has some kind of "memory" and adapts to a concentration derivative evaluated during some time step $\Delta t$.

Although the swimming directions before and after a tumble are correlated, with a mean angle between both directions of $\sim 60^{\circ}[1,19]$, we neglect this fact and in our model the bacterium randomly chooses a new orientation angle $\theta$ after each run.

\subsection{Oxygen dynamics}

In this work we consider oxygen as the only chemoattractant in the bacteria's environment. The dynamics of the oxygen concentration $c$ are described by the following equation:

$$
\partial_{t} c+\mathbf{u} \cdot \nabla c-D_{c} \Delta c=-g(c, B),
$$

where $D_{c}$ is the oxygen diffusion coefficient, $\mathbf{u}$ the fluid velocity and $-g(c, B)$ is the sink term accounting for the oxygen consumption by the bacteria. This term can be written as follows:

$$
g(c, B)=\kappa f(c) \chi_{B},
$$

where $\kappa$ is the volumic consumption rate (in oxygen molecules per second per volume of bacteria), $\chi_{B}$ is the characteristic function for the "bacterial" domain, and $c \rightarrow f(c)$ modulates this rate, going to 1 when $c$ is large, but vanishing as $c$ is smaller than a critical value (in the simulations, we will use the function $f(c)=c$ for the sake of simplicity).

Notice also that, as a feedback, the motion of the bacteria can be related to the oxygen concentration as follows: bacteria which do not consume enough oxygen loose their capacity of self-propulsing [4]. Therefore, the intensity of the self-propulsion force $f_{p}$ is modulated by the so-called motility function $r(c)$ which is equal to unity at large $c$ and vanishes rapidly for $c$ below the motility cutoff $c^{*}$. 


\section{The COUPLED Fluid AND RIGID BODY PROBLEM}

\subsection{Variational formulation over constrained spaces}

Following what has been done in $[12,15]$, we establish a variational formulation of the continuous problem involving functions which are defined on the whole domain. In that way we will avoid working on the time dependent fluid domain, that would necessitate remeshing. This can be achieved by introducing rigid constraints into the functional spaces considered:

$$
\begin{aligned}
& K_{\nabla}=\left\{\mathbf{u} \in H_{0}^{1}(\Omega), \nabla \cdot \mathbf{u}=0\right\} \\
& K_{B}=\left\{\mathbf{u} \in H_{0}^{1}(\Omega), \forall i \exists\left(\mathbf{v}_{i}, \omega_{i}\right) \in \mathbb{R}^{2} \times \mathbb{R} ; \mathbf{u}(\mathbf{x})=\mathbf{v}_{i}+\omega_{i} \times\left(\mathbf{x}-\mathbf{x}_{i}\right) \text { a.e. in } B_{i}\right\} .
\end{aligned}
$$

Here $K_{\nabla}$ is the space of divergence free functions defined on $\Omega$, and $K_{B}$ is the space of functions which do not deform $B$. The latter can also be written as follows:

$$
K_{B}=\left\{\mathbf{u} \in H_{0}^{1}(\Omega), \mathbb{D}(\mathbf{u})=0 \text { a.e. in } B\right\} .
$$

The solution to the initial problem, defined on $\Omega \backslash \bar{B}$, can be extended on the whole domain $\Omega$ by a function in $K_{B}: \mathbf{u}(\mathbf{x}, \cdot)=\mathbf{v}_{i}+\omega_{i} \times\left(\mathbf{x}-\mathbf{x}_{i}\right)$ in $B_{i} \forall i$, and we still denote this extension by $\mathbf{u}$.

Let $(\mathbf{u}, p)$ be the extended solution of the problem. On the one hand we write the incompressibility equation in its usual variational form. On the other hand we multiply the momentum equation by a test function $\widetilde{\mathbf{u}}$ in $K_{B}$, integrate it by parts over $\Omega \backslash \bar{B}$, and then extend the integrals over $\Omega$ using the fact that $\nabla \cdot \mathbf{u}=0$ and $\mathbb{D}(\mathbf{u})=0$ in $K_{B}$. Finally, using the boundary conditions of the problem, we obtain the following variational formulation (see [12]):

$$
\left\{\begin{aligned}
2 \mu \int_{\Omega} \mathbb{D}(\mathbf{u}) \cdot \mathbb{D}(\widetilde{\mathbf{u}})-\int_{\Omega} p \nabla \cdot \widetilde{\mathbf{u}} & =\int_{\Omega} \mathbf{f} \cdot \widetilde{\mathbf{u}}, & & \forall \widetilde{\mathbf{u}} \in K_{B}, \\
\int_{\Omega} q \nabla \cdot \mathbf{u} & =0, & & \forall q \in L_{0}^{2}(\Omega),
\end{aligned}\right.
$$

where

$$
\mathbf{f}=\sum_{i=1}^{N}\left(\mathbf{f}_{b}^{i} \chi_{b}^{i}+\mathbf{f}_{p}^{i} \chi_{p}^{i}\right),
$$

and $L_{0}^{2}(\Omega)$ stands for the set of $L^{2}$ functions over $\Omega$ with zero mean value.

\subsection{Penalty method to enforce the rigid motion}

In the last two decades, different methods have been designed to simulate the motion of rigid bodies in a viscous fluid. Let us mention two important methods:

- A first class of methods relies on meshing the fluid domain, computing the flow in the fluid domain (which is complex because of the inclusions) and, as a consequence, evaluating the forces exerted on the particles and the velocity perturbations. The position of the inclusions is updated and the mesh has to be redefined in the new fluid domain. This method needs further computational techniques such as a ALE-type mesh displacement, before renewing the procedure for the next time step (see e.g. [11,13,16]). Although the conforming character of the mesh ensures a good accuracy in space, this type of methods has two major drawbacks: the unstructured character of the mesh rules out the possibility to use standard fast solvers and the numerical cost of dynamic mesh generation can become prohibitive. 
- Another class of approaches relies on fictitious domain-type methods, which allow us to use Cartesian grids in the whole domain. The presence of the entities in the fluid is dealt with an iterative algorithm on an auxiliary field (composed of Lagrange multipliers) which warrants the rigid motion constraint of the particles (see e.g. $[7,8]$ ). An alternative to this are penalty methods: the rigid motion constraint is obtained by relaxing a term in the variational formulation, which amounts to replace rigid zones by highly viscous ones (see $[12,23])$. As rigid velocities are polynomials of degree 1 , it is possible to take into account the rigid motion by combining the finite elements of the mesh that correspond to the rigid domain: this method is developped and studied in [20].

In this work, the penalty method will be used, mainly because of its ease of implementation.

Formulation (5) involves a solution and test functions defined in the constrained space of rigid motions on $B$. In order to relax this constraint and obtain a formulation adapted to a finite element discretization, we use a penalty method. This method has already been used in [15] to enforce the rigid motion constraint of particles in a fluid flow. It consists of considering the minimization problem over a constrained domain associated to (5) and relaxing the constraint by introducing a penalty term in the minimized functional. The added term is the following:

$$
\frac{1}{\varepsilon} \int_{B} \mathbb{D}(\mathbf{u}): \mathbb{D}(\mathbf{u})
$$

so that $\mathbb{D}(\mathbf{u})$ goes to zero when $\varepsilon$ goes to zero and $\mathbf{u}$ tends to a rigid motion in $B$.

The variational formulation obtained is:

$$
\left\{\begin{aligned}
2 \mu \int_{\Omega} \mathbb{D}\left(\mathbf{u}_{\epsilon}\right): \mathbb{D}(\widetilde{\mathbf{u}})+\frac{2}{\varepsilon} \int_{B} \mathbb{D}\left(\mathbf{u}_{\epsilon}\right): \mathbb{D}(\widetilde{\mathbf{u}})-\int_{\Omega} p_{\epsilon} \nabla \cdot \widetilde{\mathbf{u}} & =\int_{\Omega} \mathbf{f} \cdot \widetilde{\mathbf{u}}, & & \forall \widetilde{\mathbf{u}} \in H_{0}^{1}(\Omega), \\
\int_{\Omega} q \nabla \cdot \mathbf{u}_{\epsilon} & =0, & & \forall q \in L_{0}^{2}(\Omega),
\end{aligned}\right.
$$

It has been proven in [12] that, in this framework, the penalty method converges linearly in $\varepsilon$, i.e. solution of problem (6) $\mathbf{u}_{\epsilon}$ converges to solution of problem (5) $\mathbf{u}$ as $\varepsilon$ vanishes, and the convergence is of order 1 in $\varepsilon$. We refer to [18] for a detailed analysis of a scalar version of this problem, which provides an error estimate for the space-discretized problem at the order $\varepsilon+h^{1 / 2}$.

Let us look at a numerical simulation performed using the penalty method (here referred as ExP. 1, see Table 1, page 122). We have considered a square domain with one bacterium whose head $B$ is placed at $\mathbf{x}=(0.5,0.6)$ and whose orientation is such that $\theta=3 \pi / 2$. The variational formulation related to the penalty method (ensuring the rigid body constraint) allows us to compute the velocity field in the whole domain, using a finite element method: Fig. 2 and 3 highlight the influence of the self propulsion force and the rigid body constraint (note that the flow is considered to be at rest in absence of bacteria). See in particular $a$ ) the velocity field in the domain, $b$ ) the first component of the velocity field and $c$ ) the second component of the velocity field. The location of the flagellum $P$ can be deduced from the second component of the velocity field, since it matches with the high peak negative value (the propulsion force being directed to the bottom); as a response, the high peak positive value of the second component of the velocity field matches with the head $B$ of the bacterium.

\section{Time DiscRETIZATION}

We denote by $\Delta t>0$ the time step and for any function $(\mathbf{x}, t) \rightarrow f(\mathbf{x}, t)$, we denote by $f^{n}$ the approximation of $f(\cdot, n \Delta t)$. At $t=t^{n}$, the solid domain is denoted by $B^{n}=\cup B_{i}^{n}$. For $i=1 \ldots N$, the $i$ th particle is defined by the position $\mathbf{x}_{i}^{n}$ of its center of gravity at time $t^{n}$, and the orientation $\theta_{i}^{n}$ of its self-propulsion force. 

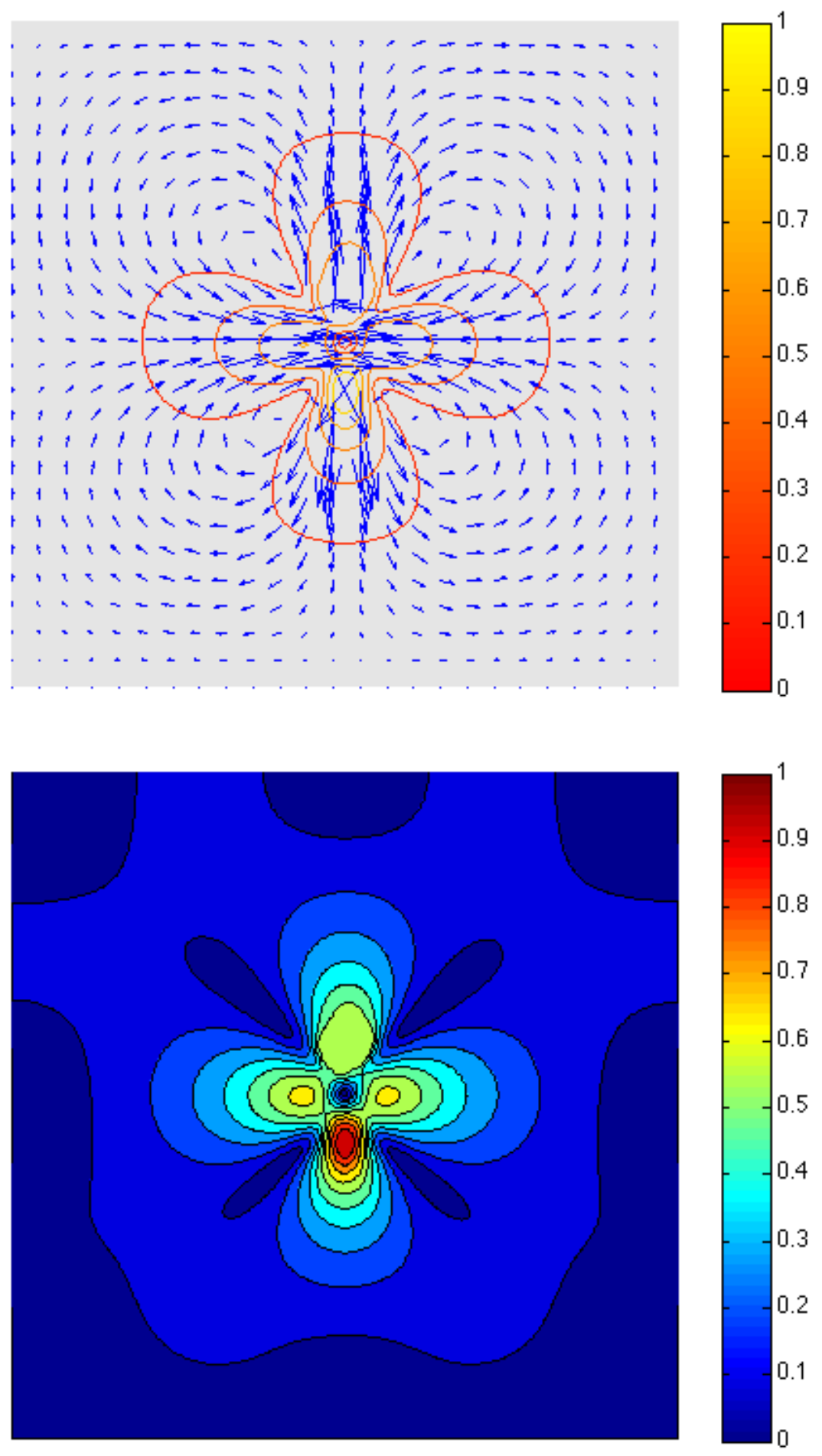

Figure 2. Full model. Streamlines (t) and norm (b) of the velocity field in a square domain with one bacteria (ExP. 1). 

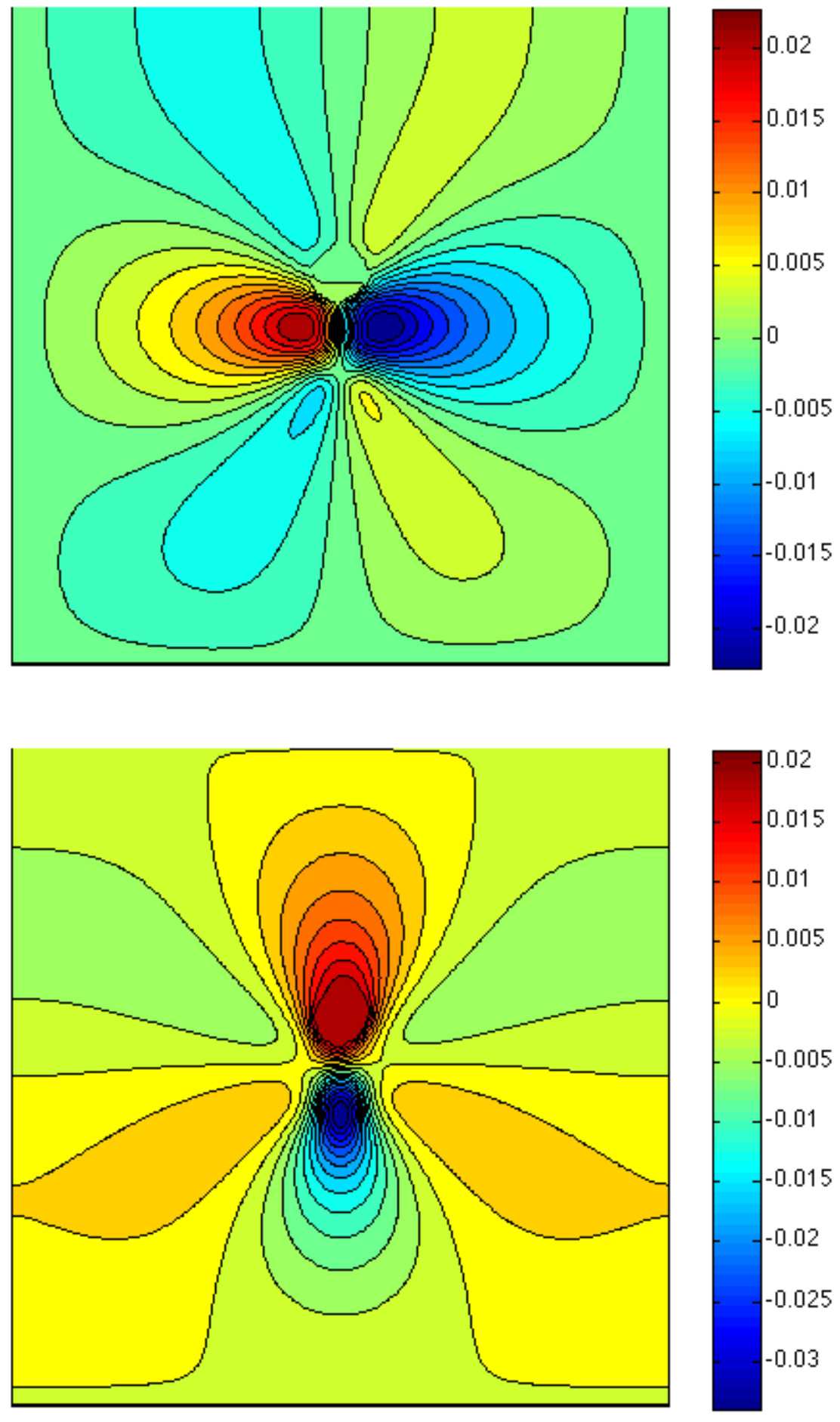

Figure 3. Full model. First component (t) and second component (b) of the velocity field in a square domain with one bacteria (ExP. 1). 


\subsection{A contact algorithm}

Assuming, as we did in our model, that particle surfaces are smooth and that Stokes model is valid at any scale, it is known that contacts are not supposed to happen (see $[6,10])$. Yet in actual simulations, collisions between particles are likely to occur. From a numerical point of view, it means that the spheres may overlap when their positions are updated after the velocity field computation. The treatment of possible overlap of the spheres is even more important in the case of dense suspensions. For that purpose we use a numerical method proposed by Maury in [17], where inelastic collisions between rigid particles are computed. It consists of projecting the velocity field onto some convex set depending on the current configuration, so that particles do not overlap. To be more precise, let us denote by $\mathbf{X}^{n}:=\left(\mathbf{x}_{i}^{n}\right)_{i=1, \ldots, N}$ the position of $N$ bacteria (more precisely, the position of their gravity centre) at time $t_{n}$. Now we define the set

$$
K\left(\mathbf{X}^{n}\right)=\left\{\mathbf{V} \in \mathbb{R}^{2 N}, D_{i j}\left(\mathbf{X}^{n}\right)+\Delta t \mathbf{G}_{i j}\left(\mathbf{X}^{n}\right) \cdot \mathbf{V} \geq 0, \forall i<j\right\}
$$

where

$$
D_{i j}\left(\mathbf{X}^{n}\right)=\left|\mathbf{x}_{i}^{n}-\mathbf{x}_{j}^{n}\right|-2 R_{b}
$$

denotes the signed distance between two spheres $B_{i}$ and $B_{j}$ and

$$
\mathbf{G}_{i j}\left(\mathbf{X}^{n}\right)=\nabla D_{i j}=\left(\ldots, 0,-\mathbf{e}_{i j}, 0, \ldots, 0, \mathbf{e}_{i j}, 0, \ldots\right), \quad \mathbf{e}_{i j}=\frac{\mathbf{x}_{j}-\mathbf{x}_{i}}{\left|\mathbf{x}_{j}-\mathbf{x}_{i}\right|}
$$

is the gradient of the distance.

In order to avoid overlapping, the following splitting procedure is proposed: in a first step, we solve the variational problem without taking into account the possible overlapping of the particles (thus defining an $a$ priori velocity of the spheres), then compute the projection of this a priori velocity onto the set of admissible velocities defined by (7). This projection is performed by an Uzawa algorithm on its saddle-point formulation.

The algorithm reads as follows: Assume that the position of the bacteria $\left(\mathbf{X}^{n}, \boldsymbol{\theta}^{n}\right)$ is known at time $t_{n}$. We aim at determining the velocity field $\mathbf{u}^{n}$ in the fluid, the motion of the bacteria $\left(\mathbf{V}^{n}, \boldsymbol{\omega}^{n}\right)$ and update the position of the bacteria $\left(\mathbf{X}^{n+1}, \boldsymbol{\theta}^{n+1}\right)$ :

i. Solve the variational formulation: find $\left(\mathbf{u}^{n}, p^{n}\right) \in H_{0}^{1}(\Omega) \times L_{0}^{2}(\Omega)$ such that

$$
\left\{\begin{array}{rlrl}
2 \mu \int_{\Omega} \mathbb{D}\left(\mathbf{u}^{n}\right): \mathbb{D}(\widetilde{\mathbf{u}})+\frac{2}{\varepsilon} \int_{B} \mathbb{D}\left(\mathbf{u}^{n}\right): \mathbb{D}(\widetilde{\mathbf{u}})-\int_{\Omega} p^{n} \nabla \cdot \widetilde{\mathbf{u}} & =\int_{\Omega} \mathbf{f}^{n} \cdot \widetilde{\mathbf{u}}, & & \forall \widetilde{\mathbf{u}} \in H_{0}^{1}(\Omega), \\
\int_{\Omega} q \nabla \cdot \mathbf{u}^{n} & =0, & \forall q \in L_{0}^{2}(\Omega) .
\end{array}\right.
$$

ii. Compute the a priori translational and angular velocity of the bacteria:

$$
\hat{\mathbf{v}}_{i}^{n}=\int_{B_{i}^{n}} \mathbf{u}^{n}(\mathbf{x}) \mathrm{d} \mathbf{x}, \quad \omega_{i}^{n}=\frac{\int_{B_{i}^{n}}\left(\mathbf{x}-\mathbf{x}_{i}^{n}\right)^{\perp} \cdot \mathbf{u}^{n}(\mathbf{x}) \mathrm{d} \mathbf{x}}{\int_{B_{i}^{n}}\left|\mathbf{x}-\mathbf{x}_{i}^{n}\right|^{2} \mathrm{~d} \mathbf{x}}
$$

iii. Compute the admissible velocity $\mathbf{V}^{n}$ as the projection of the a priori velocity $\hat{\mathbf{V}}^{n}$ :

$$
\left|\mathbf{V}^{n}-\hat{\mathbf{V}}^{n}\right|^{2}=\min _{\mathbf{V} \in K\left(\mathbf{X}^{n}\right)}\left|\mathbf{V}-\hat{\mathbf{V}}^{n}\right|^{2}
$$

iv. Update the position of the bacteria at time $t_{n+1}$ :

$$
\mathbf{X}^{n+1}=\mathbf{X}^{n}+\Delta t \mathbf{V}^{n}, \quad \boldsymbol{\theta}^{n+1}=\boldsymbol{\theta}^{n}+\Delta t \boldsymbol{\omega}^{n} .
$$


The interest in the procedure relies on the possibility to use any suitable solver for the computation of the dynamics. Contacts are handled at a second stage, without any consideration of the proper dynamics. At some point, it allows the use of any solver for the resolution of the dynamics problem: then the so-called predicted velocity field is projected onto the set of admissible velocity fields.

Let us give some comprehensive details on the contact algorithm and the effective procedure related to it. The numerical treatment of the constraint is based on an equivalent formulation of the minimization problem, as it is is treated as a saddle-point problem, by using the introduction of Lagrange multipliers:

$$
\left\{\begin{array}{l}
\text { Find }\left(\mathbf{V}^{n}, \boldsymbol{\Lambda}^{n}\right) \in \mathbb{R}^{2 N} \times \mathbb{R}_{+}^{N(N-1) / 2} \text { such that } \\
\mathcal{J}\left(\mathbf{V}^{n}, \lambda\right) \leq \mathcal{J}\left(\mathbf{V}^{n}, \boldsymbol{\Lambda}^{n}\right) \leq \mathcal{J}\left(\mathbf{V}, \boldsymbol{\Lambda}^{n}\right), \quad \forall(\mathbf{V}, \lambda) \in \mathbb{R}^{2 N} \times \mathbb{R}_{+}^{N(N-1) / 2}
\end{array}\right.
$$

with the following functional:

$$
\mathcal{J}(\mathbf{V}, \lambda)=\frac{1}{2}\left|\mathbf{V}-\hat{\mathbf{V}}^{n}\right|^{2}-\sum_{1 \leq i<j \leq N} \lambda_{i j}\left(D_{i j}\left(\mathbf{X}^{n}\right)+\Delta t \mathbf{G}_{i j}\left(\mathbf{X}^{n}\right) \cdot \mathbf{V}\right) .
$$

Notice that the number of Lagrange multipliers corresponds to the number of possible contacts. In particular, if there is no contact between particles $i$ and $j$, then $\lambda_{i j}=0$ and the Lagrange multiplier is not activated; conversely, if there is a contact between the two spheres, then $\lambda_{i j}$ may be positive and the corresponding auxiliary field allows the velocity field to satisfy the no-overlapping constraint. The approximate reaction field $\boldsymbol{\Lambda}^{n}=\left(\Lambda_{i j}^{n}\right)$ is the dual component of a solution to the associated saddle-point problem. This problem is solved by an Uzawa algorithm (see, e.g., [3]).

\subsection{Discrete tumbling}

In order to simulate tumble for each bacterium, we need to time-discretise a Poisson process with variable intensity (i.e. the frequency of tumbling $\lambda$ ). For that purpose, we set $\Delta t_{T}>0$ a fixed time-step, and we define $P_{T}$ the probability of a bacterium to tumble at least one time during $\Delta t_{T}$. This probability can be computed and depends on the values of $\Delta t_{T}$ and $\lambda$ :

$$
P_{T}\left(\lambda, \Delta t_{T}\right)=1-\exp \left(-\lambda \Delta t_{T}\right)
$$

Now, for each bacterium, the frequency of tumbling $\lambda$ varies in time following (3). Therefore, the value of $P_{T}$ also varies in time, depending on the sign of the lagrangian derivative of the concentration $\mathrm{D} c / \mathrm{D} t$ evaluated by the bacterium along its trajectory. After each time-step $\Delta t_{T}$, each bacterium performs a random reorientation (a tumble) with probability:

$$
P_{T}(c)=1-\exp \left(-\left(1-\varepsilon \phi\left(\frac{\mathrm{D} c}{\mathrm{D} t}\right)\right) \Delta t_{T}\right) .
$$

Note that with this procedure we neglect the fact that there can be more than one reorientation during $\Delta t_{T}$. But this has no influence on the result as long as we neglect correlation between the directions before and after tumbling.

\section{Numerical Results}

\subsection{Chemotactic model (oxygen-bacteria model)}

At this stage, we aim at simulating the chemotactic response of the bacteria with respect to the oxygen distribution and consumption. The fluid influence is neglected and only oxygen behaviour coupled with bacteria motion is considered. Since the penalized Stokes problem is not solved in this case, the computational cost is 
very much reduced. The CPU time is only due to contact handling and oxygen dynamics. The motion of the bacteria is obtained as follows:

- the norm of the bacteria velocity is prescribed, taken as a constant value;

- the direction of the bacteria is determined by the chemotactic response, including runs and tumbles.

- the a priori velocity is corrected through contact handling.

Fig. 4 provides results related to a numerical simulation referred as ExP. 2 (see Table 1 for the parameters). We may observe snapshots of the oxygen distribution and position of the microstructure (800 bacteria) at different times steps $(1 \cdot \Delta t, 60 \cdot \Delta t$ and $400 \cdot \Delta t)$ in a square domain. During the whole simulation, the boundary conditions are the following: a Dirichlet boundary condition $(c=1)$ is imposed on upper boundary and a homogeneous Neumann condition is imposed on the other ones. At initial time, the oxygen distribution is homogeneous in space and the bacteria have been placed randomly in the domain. After one time step (Fig. 4-a)), we observe a local consumption of oxygen, which is larger where the density of bacteria is high. After 60 time steps (Fig. 4-b)), an oxygen gradient has appeared through the conjunction of $i$ ) consumption of oxygen by the bacteria, $i$ i) oxygen supply from the boundary modelling the fluid-air interface and iii) diffusion of oxygen into the whole domain. After 400 time steps (Fig. 4-d)), most of the bacteria have migrated towards the supply boundary, following the oxygen gradient. Notice also that, at final time, the oxygen concentration far from the supply boundary has increased because of the low density of bacteria. Interestingly, we can focus on the individual behaviour of bacteria: Fig. 5 represents the trajectories of some bacteria during all the simulation (the latest position is provided by the representation of the bacteria), which reveals the succession of runs and tumbles of the bacteria, leading to an average motion directed to the upper boundary modelling the air-fluid interface.

\subsection{Full model (oxygen-bacteria-fluid model)}

Numerical tests have been performed with the full model. In the unit square domain, we have considered 400 bacteria in a Stokes flow. Chemotaxis and hydrodynamic interactions between the bacteria and the fluid are taken into account.

In this setting, the numerical simulation presented here refers to ExP. 3 (see Table 1 for the parameters). Fig. 6 provides snapshots of the oxygen distribution and position of the microstructure (400 bacteria) at different times steps $(1 \cdot \Delta t, 200 \cdot \Delta t, 500 \cdot \Delta t$ and $2000 \cdot \Delta t)$. We may observe that some bacteria have succeeded in reaching the upper supply boundary while other bacteria have been trapped at the bottom: the latter is due to the fact that we imposed a zero-velocity of the fluid at the bottom only, so that the bacteria hardly move in this region. Fig. 7 shows the streamlines of the velocity fluid (t) and the norm of the velocity field (b) in the domain at time $t=2000 \cdot \Delta t$. Fig. 8 shows the first component of the fluid velocity, the second component of the fluid velocity in the domain at time $t=2000 \cdot \Delta t$; this allows to observe the effects of the propulsion forces on the fluid velocity and, as a response, on the bacteria. Fig. 9 represents the trajectories of some bacteria: it can be observed that the motion of the fluid now has a strong influence on the trajectories of the bacteria which tend to adapt to the oxygen gradient (via the run and tumble process) but are subject to the fluid flow.

\section{REFERENCES}

[1] H.C. Berg, Random walks in biology, Princeton University Press, Princeton (1983).

[2] B.M Haines, I.S. Aranson, L. Berlyand and D.A. Karpeev, Effective viscosity of dilute bacterial suspensions: a two-dimensional model, Phys. Biol., 5 (2008)

[3] P. G. Ciarlet, Introduction à l'analyse numérique matricielle et à l'optimisation, Masson, Paris (1990).

[4] L.H. Cisneros, R. Cortez, C. Dombrowski, R.E. Goldstein, J.O. Kessler, Fluid dynamics of self-propelled microorganisms, from individual to concentrated populations, Exp Fluids, 43:737-753 (2007).

[5] R. Erban and H.G. Othmer, From individual to collective behaviour in bacterial chemotaxis, SIAM J. Appl. Math., 65(2): 361-391 (2004). 

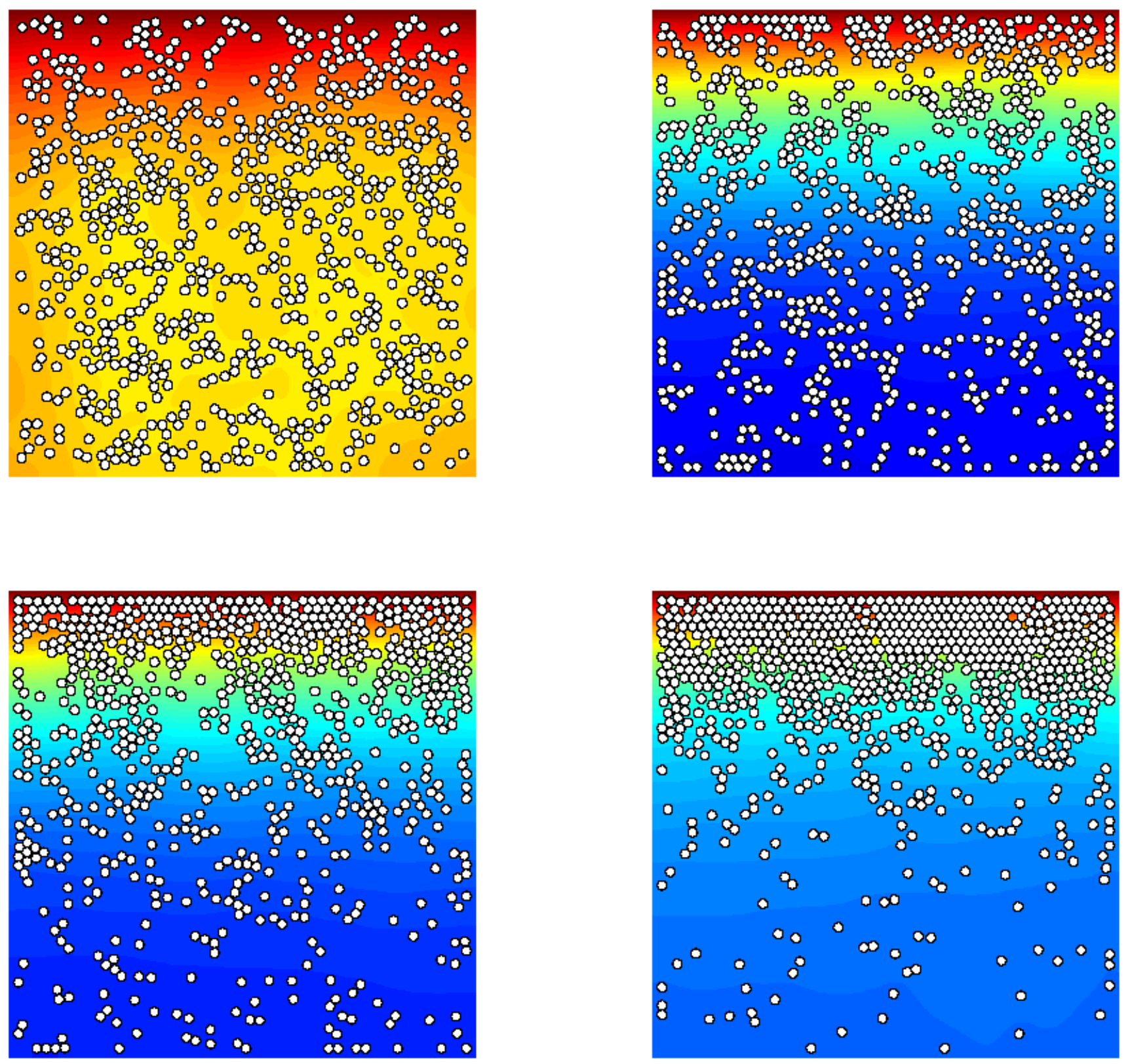

Figure 4. Chemotactic model. Oxygen distribution and position of 800 bacteria at different time steps in a square domain (ExP. 3). 

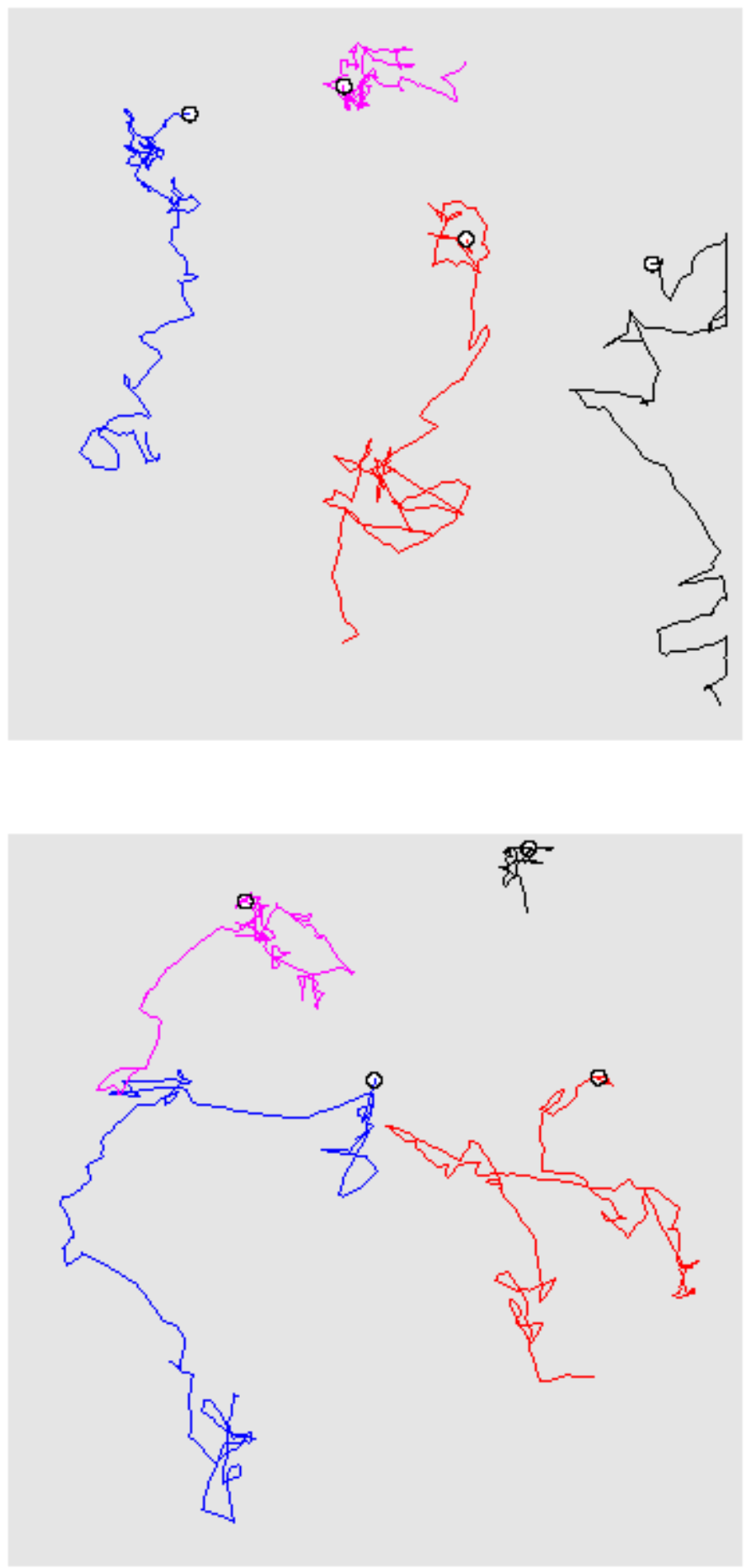

Figure 5. Chemotactic model. Random walk of some bacteria (t, b) (ExP. 3). 

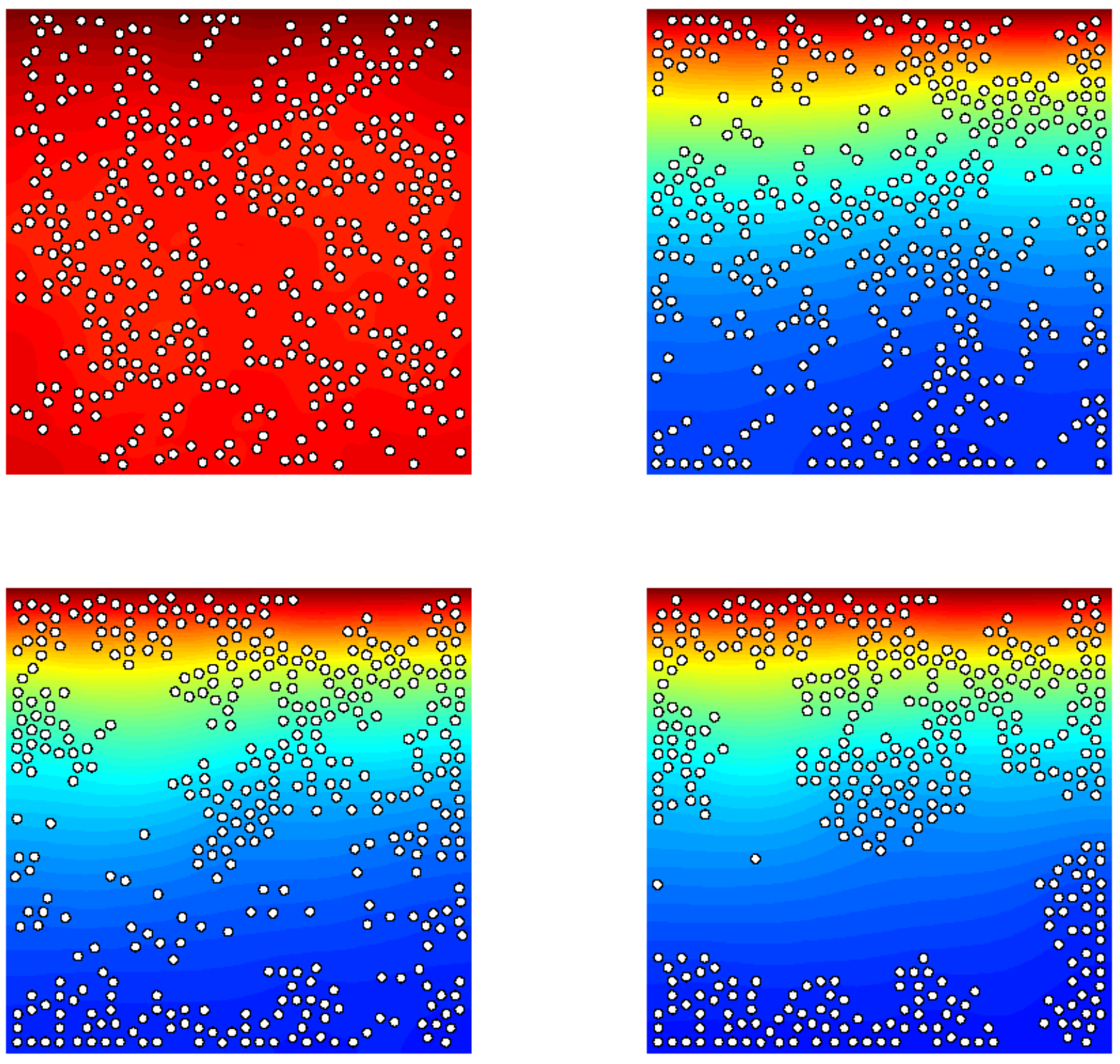

Figure 6. Full model. Oxygen distribution and position of 400 bacteria at different time steps in a square domain (ExP. 3). 

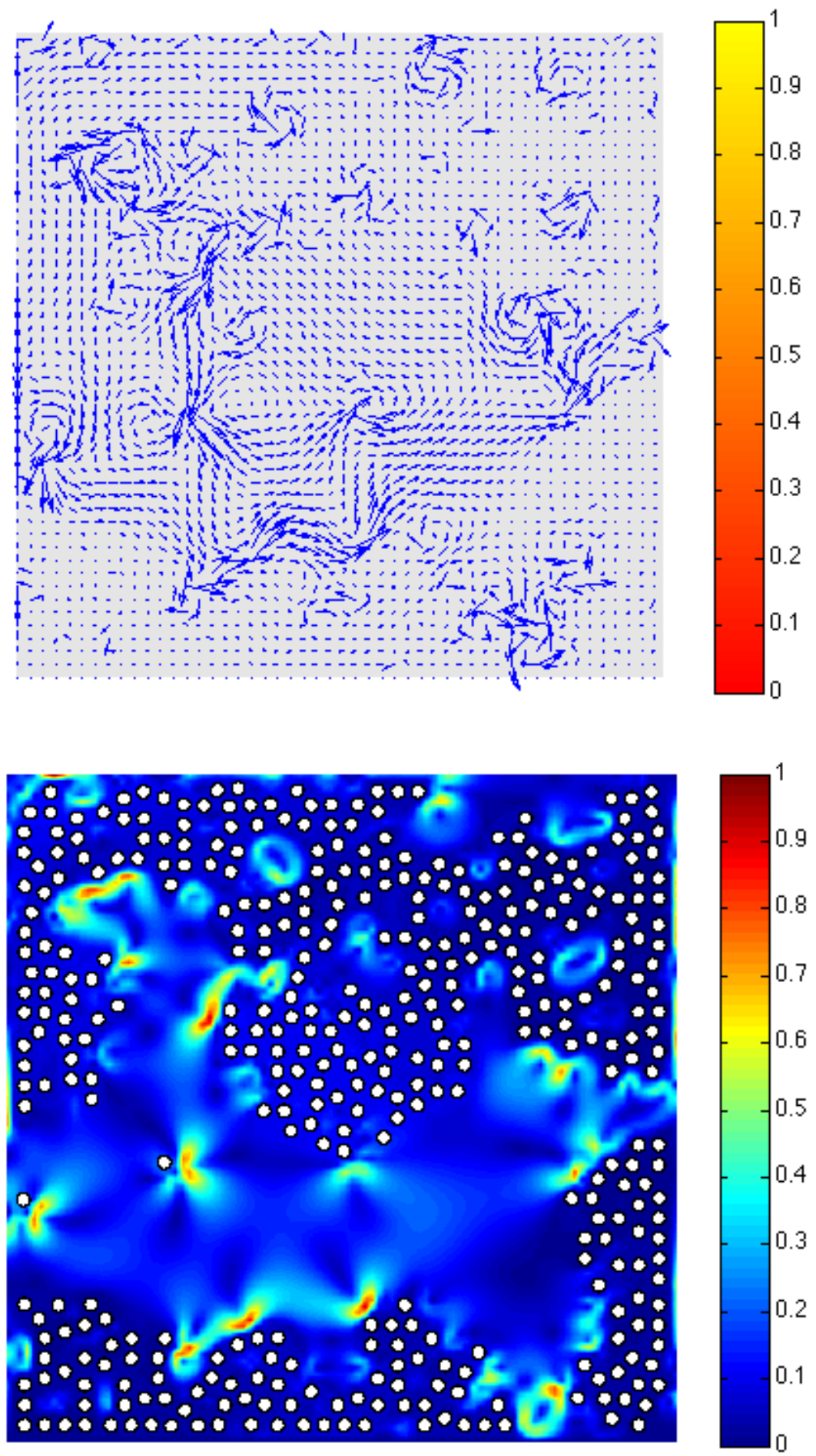

Figure 7. Full model. Streamlines (t) and norm of the velocity fluid (b) in a Stokes flow with 400 bacteria after 2000 time steps (EXP. 3). 

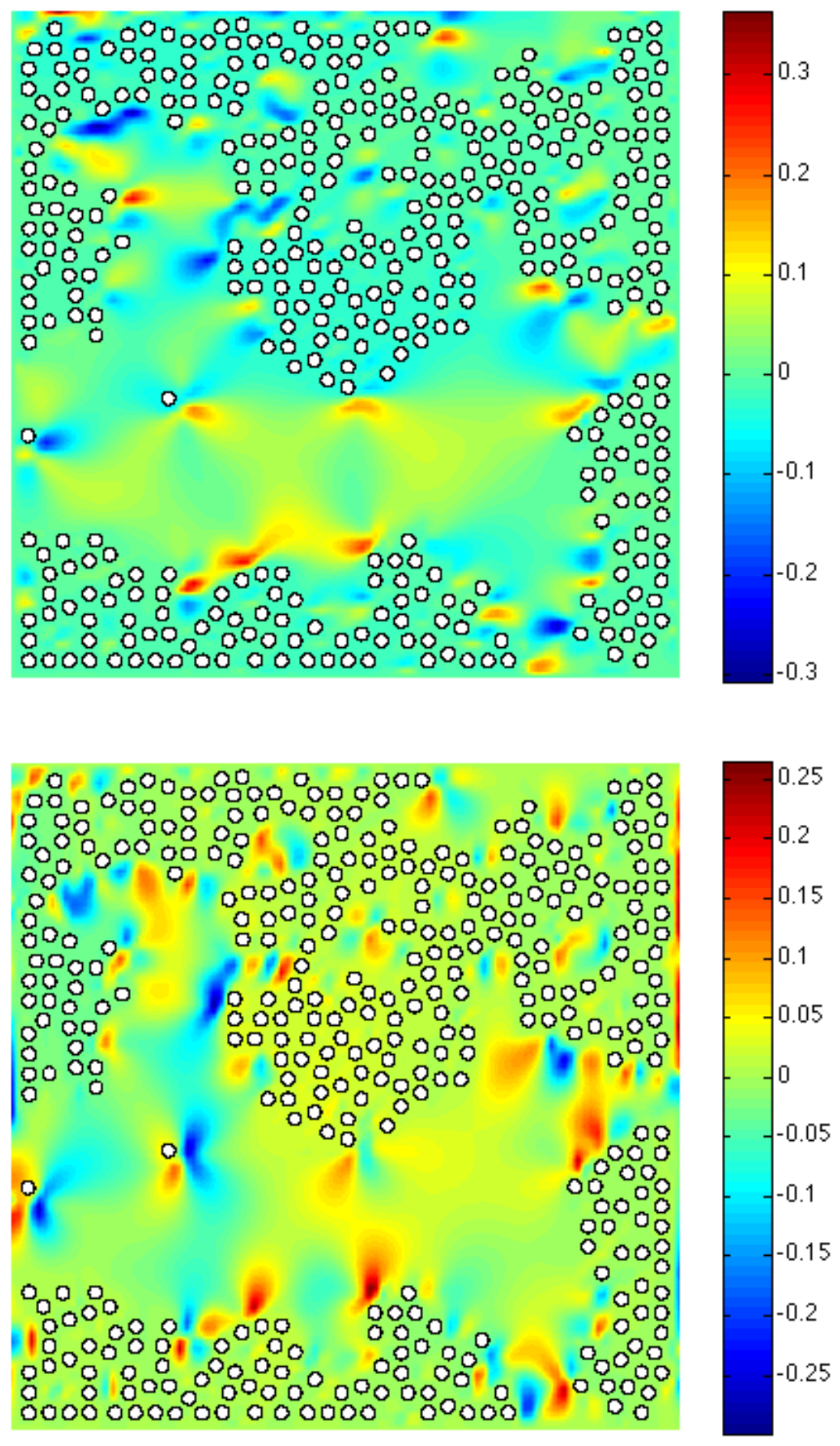

Figure 8. Full model. First component (t) and second component (b) of the velocity fluid in Stokes flow with 400 bacteria after 2000 time steps (ExP. 3). 

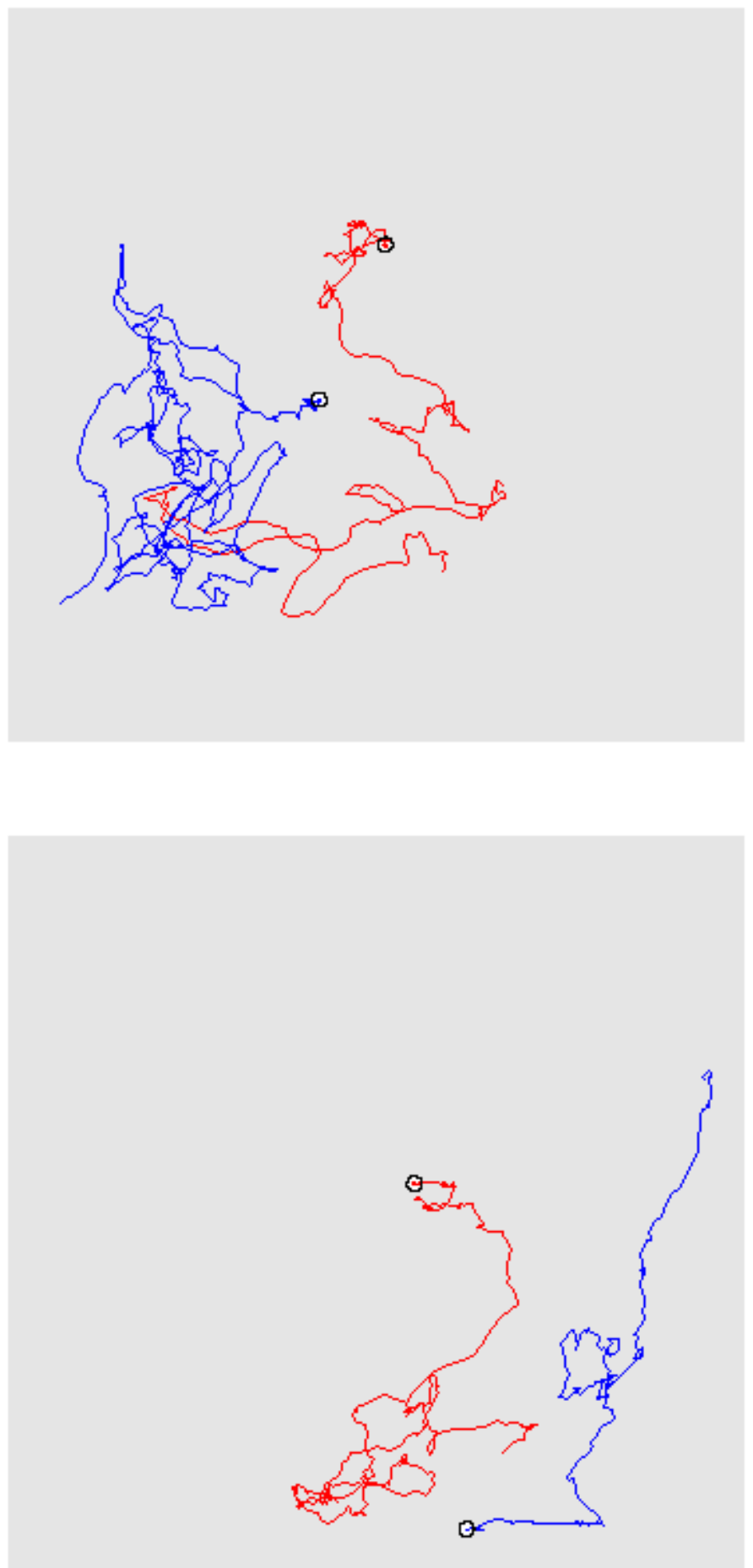

Figure 9. Full model. Random walk of some bacteria (t, b) (ExP. 3). 
ExP. 1

ExP. 2

ExP. 3

\begin{tabular}{lccc}
\hline Domain & $(0,1) \times(0,1)$ & $(0,1) \times(0,1)$ & $(0,1) \times(0,1)$ \\
\hline Mesh & & & \\
$\quad$ Vertices & 22801 & 10201 & 10201 \\
Triangles & 45000 & 20000 & 20000 \\
\hline Bacteria & & & \\
$\quad$ Number of individuals & 1 & 800 & 400 \\
$\quad$ Head radius $\left[R_{B}\right]$ & 0.0335 & 0.0101 & 0.0101 \\
Flagellum radius $\left[R_{P}\right]$ & 0.0335 & 0.0101 & 0.0101 \\
Distance head-flagellum $\left[D_{B-P}\right]$ & 0.1342 & 0.0202 & 0.0303 \\
Propulsion intensity $\left[f_{p}\right]$ & 0.3355 & 2.0000 & 5.0000 \\
Propulsion modulation $[r(c)]$ & - & 1 & 1 \\
\hline Fluid solver & & & \\
BC (lower boundary) & $\mathbf{u}=\mathbf{0}$ & - & $\mathbf{u}=\mathbf{0}$ \\
BC (other boundaries) & $\mathbf{u} \cdot \mathbf{n}=0$ & - & $\mathbf{u} \cdot \mathbf{n}=0$ \\
\hline Oxygen concentration & & & \\
Initial condition & - & $c \equiv 1$ & $c \equiv 1$ \\
BC (upper boundary) & - & $c=1$ & $c=1$ \\
BC (other boundaries) & - & $\nabla c \cdot \mathbf{n}=0$ & $\nabla c \cdot \mathbf{n}=0$ \\
Diffusion parameter $\left[D_{c}\right]$ & - & 39.00 & 0.50 \\
Consumption rate $[\kappa]$ & - & $c$ & 98.00 \\
Consumption function $[f(c)]$ & & & $c$
\end{tabular}

TABLE 1. Parameters for the numerical simulations

[6] D. Gérard-Varet, M. Hillairet, Regularity Issues in the Problem of Fluid Structure Interaction, to appear in Arch. Rational Mech. Anal.

[7] R. Glowinski, T. W. Pan, T. I. Hesla, D. D. Joseph \& J. Périaux, A fictitious domain approach to the direct numerical simulation of incompressible viscous flow past moving rigid bodies: application to particulate flow, J. Comp. Phys. 169, 363-427 (2001).

[8] R. Glowinski, Finite element methods for incompressible viscous flow, In: Handbook of Numerical Analysis, Vol. IX, P. G. Ciarlet and J.-L. Lions eds., Ed. North-Holland, Amsterdam (2003).

[9] F. Hecht, A. Le Hyaric, K. Ohtsuka, and O. Pironneau, Freefem++, finite elements software http://www.freefem.org/ff++/.

[10] M. Hillairet, Lack of collision between solid bodies in a 2D constant-density incompressible flow, Communications in Partial Differential Equations 32: 1345-1371 (2007).

[11] H. H. Hu, Direct simulation of flows of solid-liquid mixtures, Int. J. Multiphase Flow 22 (2), 335-352 (1996).

[12] J. Janela, A. Lefebvre, B. Maury, A penalty method for the simulation of fluid-rigid body interaction, ESAIM: Proc., 1:115-123 (2005).

[13] A. A. Johnson \& T. E. Tezduyar, Simulation of multiple spheres falling in a liquid-filled tube, Computer Methods in Applied Mechanics and Engineering, 134 (3), 351-373 (1996).

[14] E. Lauga and T.R. Powers, The hydrodynamics of swimming microorganisms, Rep. Prog. Phys., 72 (2009)

[15] A. Lefebvre, Fluid-particle simulations with Freefem++, ESAIM: Proc., 18:120-132 (2007).

[16] B. Maury, Direct simulations of 2D fluid-particle flows in biperiodic domains, Journal of Computational Physics 156, 325-351 (1999).

[17] B. Maury, A time-stepping scheme for inelastic collisions, Numerische Mathematik, 102(4):649-679 (2006).

[18] B. Maury, Numerical Analysis of a Finite Element / Volume Penalty Method, SIAM J. Numer. Anal. Volume 47, Issue 2, pp. 1126-1148 (2009).

[19] J.T. Locsei, T.J. Pedley, Run and Tumble in Chemotaxis in a Shear Flow; The Effect of Temporal Comparisons, Persistence, Rotational Diffusion, and Cell Shape, Bulletin of Mathematical Biology, 71:1089-1116 (2009). 
[20] J. San Martín, J.-F. Scheid, T. Takahashi \& M. Tucsnak, Convergence of the Lagrange-Galerkin method for the equations modelling the motion of a fluid-rigid system, SIAM J. Numer. Anal., 43 (4), 1539-1571, (2005).

[21] L. Turner, W.S. Ryu, H.C. Berg, Real-time imaging of fluorescent flagellar filaments, J. Bacteriol., 182(10):2793-2801 (2000).

[22] I. Tuval, L. Cisneros, C. Dombrowski, C. W. Wolgemuth, J.O. Kessler, Bacterial swimming and oxygen transport near contact lines, Proceedings of the National Academy of Sciences (USA) 102, 2277-2282 (2005).

[23] S. Vincent, J. P. Caltagirone, P. Lubin \& T. N. Randrianarivelo, An adaptative augmented Lagrangian method for threedimensional multimaterial flows, Computers and Fluids 33, 1273-1289 (2004). 